\title{
$\mathbb{A}$ Economics Bulletin
}

\section{Volume 37, Issue 3}

\section{Staying when the Going Gets Tough: The Equivalent Predictions of Option and Search Theory on Migration During Economic Downturns}

\author{
John Gardner \\ University of Mississippi
}

\author{
Joshua R Hendrickson \\ University of Mississippi
}

\begin{abstract}
We show that both option and search theory suggest that workers should be less likely to migrate out of labor markets in which there is greater uncertainty about future economic opportunities. The intuition for this result is that, the more volatile the labor market, the greater the likelihood of such opportunities improving in the future. This result weakens the standard prediction that workers should migrate out of markets experiencing relative economic downturns.
\end{abstract}

We thank an anonymous referee for helpful comments.

Citation: John Gardner and Joshua R Hendrickson, (2017) "Staying when the Going Gets Tough: The Equivalent Predictions of Option and Search Theory on Migration During Economic Downturns", Economics Bulletin, Volume 37, Issue 3, pages 2102-2110

Contact: John Gardner - jrgardne@olemiss.edu, Joshua R Hendrickson - jrhendr1@olemiss.edu.

Submitted: July 10, 2017. Published: September 27, 2017. 


\section{Introduction}

In traditional investment models of migration, workers move if the benefits of doing so exceed the costs (these models date back at least to Sjaastad, 1962). According to this view, workers are more likely to migrate away from locations experiencing declining economic conditions. Here, we show that uncertainty about future economic conditions in the current location mutes this prediction, intuitively by increasing the expected future payoff of remaining there. Importantly, we show that this result is compatible with an option theory of migration, in which workers choose the optimal time to exercise the option to migrate, and a search theory in which workers continue to search for employment in their present location as long as the relative benefit of searching exceeds the cost of migrating.

Though our parsimonious option and search models are largely standard (see Dixit and Pindyck 19.94 and Stokey 200.9 for option models and Mortensen and Pissarides 1999. for search models; our search model is a simplification of the setup used by Black (1995), they differ from previous such models of migration in a few important ways. While many models focus on wages, we assume that workers care about the overall quality of labor markets (which may include non-pecuniary job aspects such as benefits, scheduling flexibility, the stability of the firm, and the probability of employment itself). Though other studies have considered uncertainty about economic conditions across locations (see Burda 1993 and O'Connell 1997 in the option context and Rogerson 1982, Gordon and Vickerman 1982, Maier 1985 and Berninghaus and Seifort-Vogt L9.91 in the search context), our models focus on the effect of uncertainty about future opportunities in the current location on the decision to migrate to an alternative location in which economic conditions are known. ${ }^{\text {m }}$

In the remainder of this paper, we show that both option and search views of the migration decision predict that, other things equal, workers will be less likely to migrate away from locations with greater uncertainty about future economic opportunities. Our purpose in this paper is to show that this prediction can be motivated by both option and search theory (we provide extensive empirical support for this prediction in Gardner and Hendrickson (2016)). However, in Figure $\square$ we motivate our theoretical argument empirically using evidence from 2010 American Community Survey samples of working-age men with at least some college (Ruggles et al., 2015). Showing that volatility and outmigration are negatively related, the first panel plots rates of migration out of metropolitan areas against residuals from a regression of the standard deviation of unemployment on the unemployment rate (within metropolitan areas).

We caution that if the level and volatility of quality are correlated, other things will not be equal, and rates of migration away from locations with relatively volatile quality may not be manifestly lower (before controlling for the mean of quality, that is). The second panel of the figure, which replaces the residual standard deviation of unemployment with the standard deviation itself, illustrates this. The remaining panels of the figure show how our argument attenuates the prediction of the standard model. Only after controlling for the standard deviation of unemployment (panel 3) is there a clear positive relationship between

\footnotetext{
${ }^{1}$ Though we assume opportunities are known in the alternative location mainly for simplicity, this assumption can be justified by noting that it is relative quality that workers care about. This assumption also allows us to derive testable predictions about migration in terms of the quality of workers' current locations, which is observable (unlike that for an unknown set of alternative locations).
} 

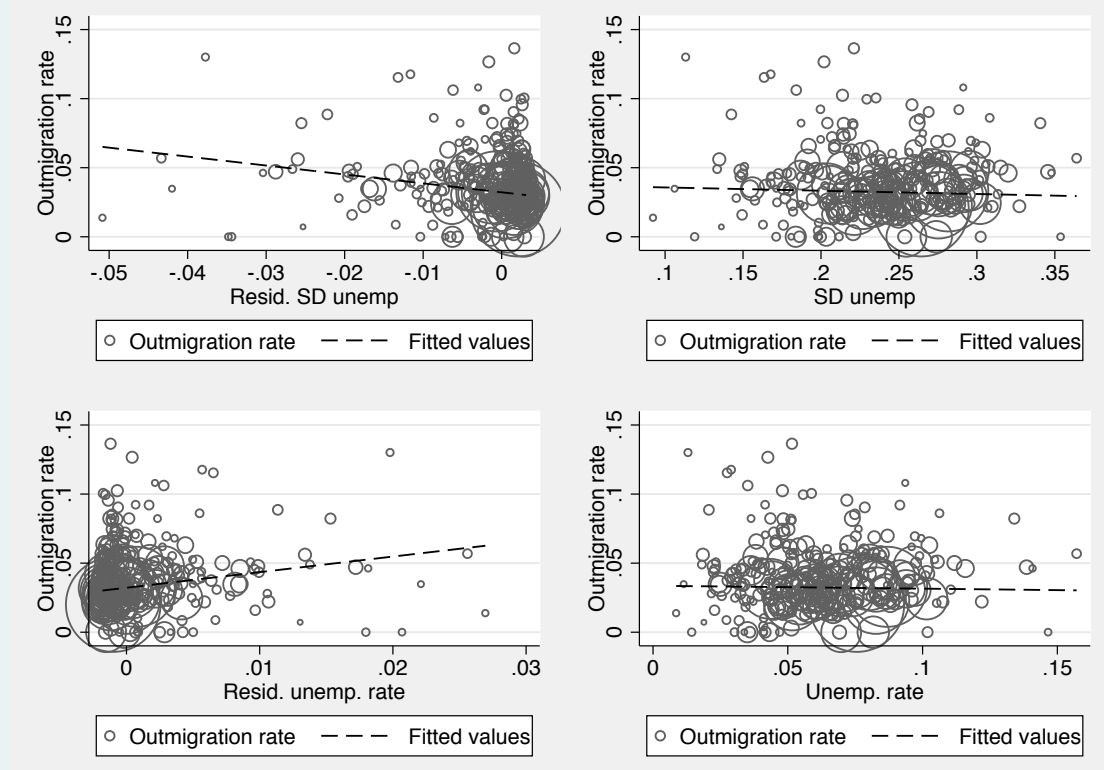

Figure 1: Outmigration rates and the volatility and level of unemployment. Metropolitan areas plotted in proportion to their size.

the unemployment rate and outmigration.

\section{The option view}

Let $q(t)$ denote the quality in the local labor market that the individual can observe. Suppose that an individual receives an instantaneous payoff that is linear in the quality of the labor market. The individual is aware of an opportunity in a different location that pays a perpetual dividend, $w$. However, moving entails a fixed cost, F. Finally, the quality of the labor market follows a geometric Brownian motion:

$$
\frac{d q}{q}=\mu d t+\sigma d z
$$

where $\mu<0$ is the expected rate of change in labor market quality, $\sigma$ is the conditional standard deviation, $d z$ is an increment of a Wiener process (i.e. $d z=\epsilon \sqrt{d t}$, where $\epsilon$ is drawn from a standard normal distribution), and $q(0)=q_{0}$.

Let $V\left(q, q^{*}\right)$ denote the value to the worker in the current location given the current state, $q$, and the threshold for labor market quality at which an individual will move, $q^{*}$. Assuming that $q_{0}>q^{*}$, the value of the option to move can be written $\operatorname{as}^{\square}$

$$
V\left(q, q^{*}\right)=\max _{q^{*}} \underbrace{\left(\frac{q}{q^{*}}\right)^{\beta}}_{\text {Stochastic Discount Factor }} \times \underbrace{\left(\frac{w}{r}-F-\frac{q^{*}}{r-\mu}\right)}_{\text {Value of Option at Exercise }}
$$

\footnotetext{
${ }^{2}$ For the derivation of this expression, see the Appendix.
} 
where $r$ is the discount rate, $w / r$ is the present value of the perpetual dividend one receives when moving, $q^{*} /(r-\mu)$ is the present value of the payoff the worker would get in the present location if he or she never moved, and $\beta<0$ is a function of the time series properties of labor market quality shown in equation (四) and the discount rate. Note that the individual faces a trade-off in determining the threshold for labor market quality. If the individual chooses a low threshold, then the value of the option at exercise is larger than it would be with a high threshold. However, a lower threshold implies that the individual will, on average, have to wait longer to move. This effect reduces the present discounted value of the option to move at exercise by reducing the stochastic discount factor. As a result of this trade-off, the individual's problem is to choose $q^{*}$ to maximize the value of the option to move. The intuition is as follows. A lower threshold, $q^{*}$, increases the value of the option at exercise, but reduces the present value of the option (because it tends to take longer to hit a lower threshold). Thus, the worker must choose $q^{*}$ to optimally balance this trade-off. The first-order condition is

$$
q^{*}=\frac{\beta}{\beta-1}(r-\mu)\left(\frac{w}{r}-F\right) .
$$

As shown in the Appendix, $\beta$ is a function of the volatility of labor market quality in the current location, $\sigma$. As a result, $q^{*}$ is also a function of $\sigma$. Since comparative statics are difficult to obtain analytically, we plot the relationship between $q^{*}$ and $\sigma$ in Figure 1 using a numerical example. As shown, when $\sigma$ increases, the individual is willing to tolerate a lower level of labor market quality. The intuition is that greater volatility in the quality of the labor market implies a larger magnitude of positive shocks to quality, which are sufficient to keep the individual in the market at lower levels of labor market quality than they would otherwise.

\section{The search view}

In this section, we compare the preceding results with those from a simple job-search model. Though they answer the same question, our option and search models differ in several ways. While our option-theory approach models the evolution of labor market quality over time, equilibrium search models typically assume that workers face stationary environments. Accordingly, in this section we compare the job search and migration behavior of workers in labor markets that have adjusted to the same mean of quality, but differ in the volatility of the quality of job offers. While our option approach assumes that the quality of a labor market applies to all workers, heterogenous outcomes is a cornerstone of job search theory. Thus, our search model assumes that the quality of job offers differs between workers within local labor markets and that the equilibrium quality of the job offers that workers accept varies across local labor markets. In contrast to our option model, in which workers choose the quality $q^{*}$ at which they leave their local labor market in favor of the outside option, in our search model, they first decide the quality at which they are willing to stop searching within a given local labor market, then decide whether they should remain in that market or search for employment elsewhere.

Suppose that, for every period of time that they search for a job, unemployed workers in a particular labor market receive job offers of random quality $q$. To model variation across local labor markets in the volatility of quality, assume that $q$ is drawn from from a mean-zero 


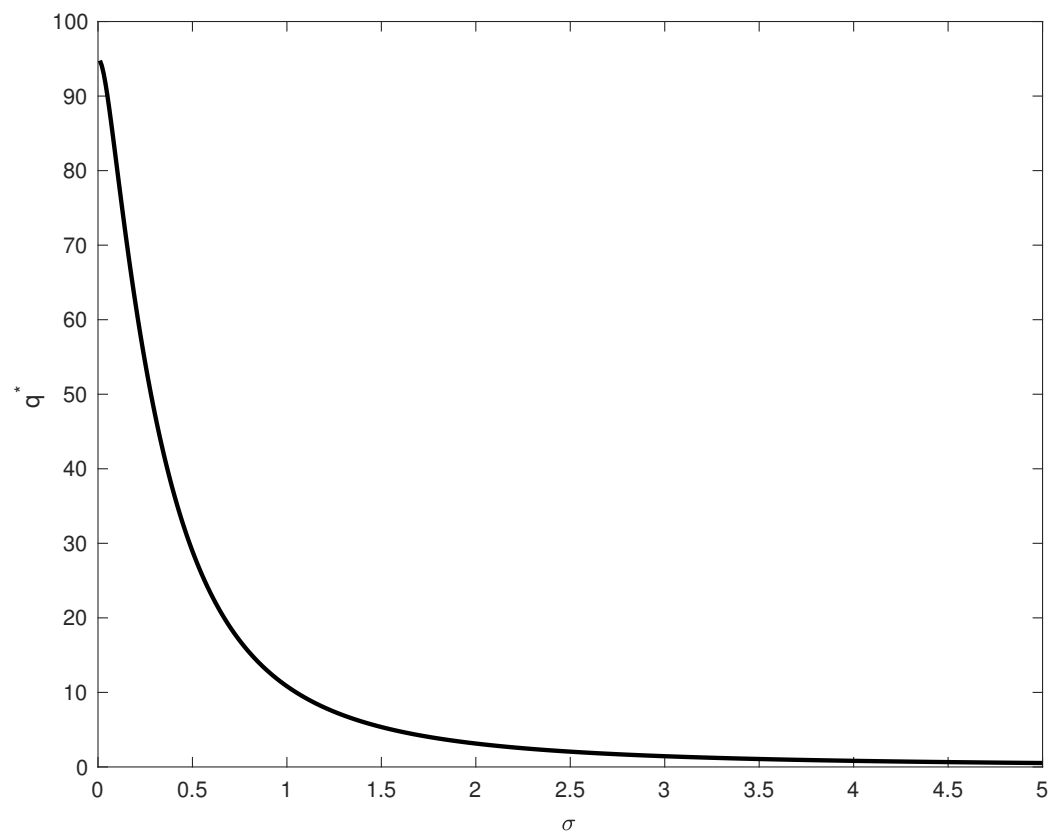

Figure 2: The figure plots the relationship between $q^{*}$ and $\sigma$ using a numerical example. For the numerical example, $w=100, F=100, \mu=-0.01$, and $r=0.05$.

normal distribution with variance $\sigma^{2}$. Our model explores how the job search and migration decisions of workers in markets with identical mean quality change with the volatility, $\sigma$, of the quality of the labor market.

Given an offer of quality $q$, the worker can either accept the job (and receive lifetime quality $q$ ) or continue to search at $\operatorname{cost} c>0$. Thus, the value $V$ of search is

$$
V=E \max (q, V)-c
$$

Let $r$ be the reservation quality at which a worker will accept a job and discontinue search and let $\Phi$ and $\phi$ denote the standard normal CDF and PDF. With probability $1-\Phi(r / \sigma)$ the worker accepts a job that offers expected quality $E(q \mid q \geq r)=\int_{r}^{\infty} q \phi(q / \sigma) /\{\sigma[1-$ $\Phi(r / \sigma)]\} d q$; with probability $\Phi(r / \sigma)$ the worker continues to search, which is valued at $V$; the cost $c$ is incurred in either case. Thus, value of search can be written

$$
V=\int_{r}^{\infty} q \frac{1}{\sigma} \phi\left(\frac{q}{\sigma}\right) d q+\Phi\left(\frac{r}{\sigma}\right) V-c .
$$

Because the worker should accept any job offer with quality in excess of that associated with searching, it must be that $r=V$. Collecting terms and rearranging, the reservation quality must therefore satisfy

$$
r=\int_{r}^{\infty} q \frac{\phi(q / \sigma) / \sigma}{1-\Phi(q / \sigma)} d q-\frac{c}{1-\Phi(r / \sigma)}=E(q \mid q \geq r)-\frac{c}{1-\Phi(r / \sigma)}
$$


To provide an economic interpretation of (5), note that (bringing the second term inside the integral) it can also be written $r=E(q-c \mid q \geq r)$, showing that the optimal reservation quality is the expected net benefit of accepting a job.

Under the assumption that $q \sim N(0, \sigma)$, we can also use standard results on the moments of the truncated normal distribution (see Heckman and Honoré, 1990, Appendix A) to express (可) as

$$
r=E(q \mid q \geq r)-\frac{c}{1-\Phi(r / \sigma)}=\sigma \lambda\left(\frac{r}{\sigma}\right)-\frac{c}{1-\Phi(r / \sigma)},
$$

where $\lambda(x)=\phi(x) /[1-\Phi(x)]>0$ for $x \in \mathbb{R}$ and, for future reference, $\lambda^{\prime}(x)=\lambda(x)[\lambda(x)-x]$.

Implicitly differentiating (6) with respect to $\sigma$ gives

$$
\begin{aligned}
r^{\prime} & =\lambda\left(\frac{r}{\sigma}\right)+\left\{\sigma \lambda\left(\frac{r}{\sigma}\right)\left[\lambda\left(\frac{r}{\sigma}\right)-\frac{r}{\sigma}\right]-\frac{c \phi(r / \sigma)}{[1-\Phi(r / \sigma)]^{2}}\right\}\left(\frac{\sigma r^{\prime}-r}{\sigma^{2}}\right) \\
& =\lambda\left(\frac{r}{\sigma}\right)+\lambda\left(\frac{r}{\sigma}\right)\left[\sigma \lambda\left(\frac{r}{\sigma}\right)-r-\frac{c}{1-\Phi(r / \sigma)}\right]\left(\frac{\sigma r^{\prime}-r}{\sigma^{2}}\right) \\
& =\lambda\left(\frac{r}{\sigma}\right)>0,
\end{aligned}
$$

where the first equality above follows from the definition of $\lambda$ and the second from condition (6). This envelope result states that, though an increase in $\sigma$ increases the value of search both by changing the shape of the quality distribution and by changing the reservation quality itself, the second effect disappears when the reservation quality is chosen optimally. An increase in the volatility of labor-market quality therefore increases the likelihood of receiving a high-quality job offer in the future, and consequently the reservation quality at which workers stop searching.

We illustrate this result numerically in Figure B. The figure plots the function $E(q \mid q \geq$ $r)-c /[1-\Phi(r / \sigma)]$ for a series of mean-zero normal distributions with increasing standard deviations. The equilibrium reservation qualities, which correspond to the intersections of these functions and the 45-degree line, are monotonically increasing in the volatility, $\sigma$, of the labor market.

To close the model, suppose that workers have the option of migrating to an outside labor market offering reservation quality $r^{0}$ at a random cost $m$. If $m$ has distribution function $F$ and density $f$, then the probability that a worker migrates is $P\left(r^{0}-r>m\right)=F\left(r^{0}-r\right)$. By the preceding result,

$$
\frac{d P\left(r^{0}-r>m\right)}{d \sigma}=-f\left(r^{0}-r\right) r^{\prime}<0 .
$$

Thus, an increase in the volatility of the labor market increases the likelihood that a worker remains there.

\footnotetext{
${ }^{3}$ Alternatively, (国) can be expressed $E[(q-r) 1(q \geq r)]=c$, where $1(\cdot)$ is the indicator function-that is, the optimal reservation quality equates the expected benefit of a job offer (which is either $q-r$ or zero, depending on whether the offer is accepted) with the cost of searching.

${ }^{4}$ Though we abstract away from differences in mean quality, it is straightforward to show that if $q \sim$ $N\left(\mu, \sigma^{2}\right), r$ is increasing in mean quality $\mu$. In this case, $\left.r=\mu+\sigma \lambda[(r-\mu) / \sigma)\right]-c /\{1-\Phi[(r-\mu) / \sigma]\}$, so that $d r / d \mu=1-\lambda^{\prime}[(r-\mu) / \sigma]+\sigma^{-1} c \phi[(r-\mu) / \sigma] /\{1-\Phi[(r-\mu) / \sigma]\}>0$, where the inequality follows because $c>0$ by assumption and because $0<\lambda^{\prime}<1$ (Heckman and Honoré, 1990).

${ }^{5}$ Our assumption that there is only one alternative labor market is for simplicity only; it is straightforward to extend this result to the case of multiple outside markets.
} 


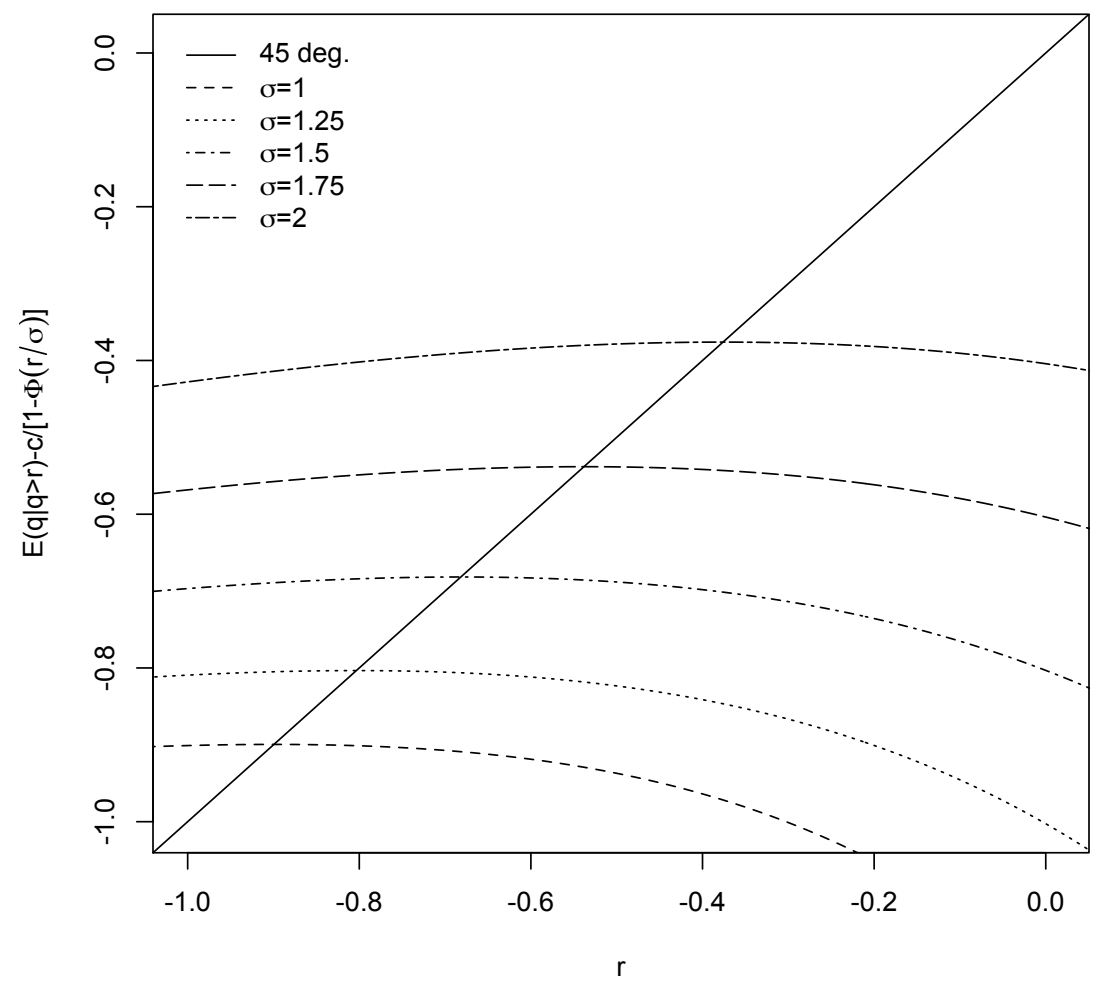

Figure 3: Equilibrium reservation quality as an implicit function of volatility. Reservation qualities correspond to the intersections of $E(q \mid q \geq r)-c /[1-\Phi(r / \sigma)]$ and the 45-degree line (for different values of $\sigma$ ).

\section{Conclusion}

Using both option and search approaches, we augment the investment theory of migration to show that, other things equal, workers should be less likely to migrate away from more volatile labor markets, softening the prediction of the traditional theory. Its compatibility with both option and search theory lends credibility to our main result, which also has implications for our understanding of migration behavior during economic downturns. While the empirical analysis presented here and in Gardner and Hendrickson (2016) focuses on internal migration, our theoretical results can also be applied to foreign and (as an anonymous referee has noted) return migration; though beyond the scope of this paper, applying the theory to those settings is a promising topic for further research.

\section{References}

Black, Dan A. (1995) "Discrimination in an Equilibrium Search Model" Journal of Labor Economics 13 (2), 309-334. 
Berninghaus, Siegfried and Hans G. Siefort-Vogt (1991) International Migration Under Incomplete Information Berlin: Springer Verlag.

Burda, Michael C. (1993) "The Determinants of East-West German Migration" European Economic Review 37, 452-461.

Dixit, Avinash K. and Robert S. Pindyck (1994) Investment Under Uncertainty, Princeton: Princeton University Press.

Gardner, John and Josh Hendrickson (2016) "If I Leave Here Tomorrow: An Option View of Migration When Labor Market Quality Declines" Working paper, http://papers.ssrn.com/sol3/papers. cfm?abstract_id=2835687.

Gordon, Ian R. and Roger W. Vickerman (1982) "Opportunity, Preference and Constraint: An Approach to the Analysis of Metropolitan Migration" Urban Studies 19, 247 - 261.

Heckman, James J, and Bo E Honoré (1990) "The Empirical Content of the Roy Model" Econometrica 58 (5), 1121-49.

Maier, Gunther (1985) "Cumulation, Causation and Selectivity in Labor Market Oriented Migration Caused by Imperfect Information." Regional Studies 19, 231-241.

Mortensen, Dale T. and Christopher A. Pissarides (1999) "New developments in models of search in the labor market" Handbook of Labor Economics 3, 2567-2627.

O'Connell, Paul G. (1997) "Migration Under Uncertainty: 'Try Your Luck' or 'Wait and See", Journal of Regional Science 37 (2), 331-347.

Rogerson, Peter A. (1982) "Spatial Models of Search" Geographical Analysis 14, 217-228.

Steven Ruggles, Katie Genadek, Ronald Goeken, Josiah Grover, and Matthew Sobek. 2015. Integrated Public Use Microdata Series: Version 6.0 [dataset]. Minneapolis: University of Minnesota, 2015. http://doi.org/10.18128/D010.V6.0.

Sjaastad, Larry A. (1962) "The Costs and Returns of Human Migration" The Journal of Political Economy 70 (5):, 80-93.

Stokey, Nancy L. (2009) The Economics of Inaction, Princeton: Princeton University Press.

\section{Appendix}

The value of option to move is equal to the present value of the perpetual dividend from moving less the fixed cost of moving and the present value of the opportunity cost of migration. Let $V\left(q, q^{*}\right)$ denote the value of the option to migrate as a function of labor market quality in the current market, $q$, and the quality threshold for moving, $q^{*}$. It follows that

$$
V\left(q, q^{*}\right)=\max _{q^{*}} \underbrace{B(q)}_{\text {Stochastic Discount Factor }} \times \underbrace{\left(\frac{w}{r}-F-\frac{q^{*}}{r-\mu}\right)}_{\text {Option Value at Exercise }}
$$


where $B(q)$ is a stochastic discount factor and the remaining terms are defined in Section 2 of the text.

Note that this expression is identical to equation (Z) with the exception of the fact that here, we have written the stochastic discount factor as an implicit function of labor market quality in the current location and the threshold for moving. Thus, what we need to do is show that $B(q)=\left(q / q^{*}\right)^{\beta}$. To do so, consider a discrete time period of length $\Delta t$. The stochastic discount factor has the following recursive representation:

$$
B(q, t)=\frac{1}{1+\rho \Delta t} E\left[B\left(q^{\prime}, t+\Delta t\right)\right]
$$

where $r$ is the discount rate. Multiplying both sides by $1+\rho \Delta t$ and re-arranging yields:

$$
r B(q, t) \Delta t=E\left[B\left(q^{\prime}, t+\Delta t\right)-B(q, t)\right] .
$$

Dividing both sides by $\Delta t$ and taking the limit as $\Delta t \rightarrow 0$ yields

$$
r B(q)=\frac{1}{d t} E d B
$$

where $d B=\lim _{\Delta t \rightarrow 0} E\left[B\left(q^{\prime}, t+\Delta t\right)-B(q, t)\right]$. Using Ito's Lemma this can be re-written as

$$
r B(q)=\frac{1}{d t} E\left[\frac{\partial B}{\partial q} d q+\frac{1}{2} \frac{\partial^{2} B}{\partial q^{2}}(d q)^{2}\right]
$$

Substituting equation (四), taking expectations, and distributing $d t$, this can be re-written as

$$
\frac{1}{2} \sigma^{2} q^{2} \frac{\partial^{2} B}{\partial q^{2}}+\mu q \frac{\partial B}{\partial q}-r B(q)=0 .
$$

Let's guess that the solution to this equation is given as $B(q)=A q^{\beta}$. Plugging this into the equation above, we can verify that this is a solution if $\beta$ is a solution to the following quadratic equation:

$$
\frac{1}{2} \sigma^{2} \beta^{2}+\left(\mu-\frac{1}{2} \sigma^{2}\right) \beta-r=0
$$

Since there are two possible values of $\beta$ the second-order differential equation (ए2) is a homogeneous linear equation, the solution can be written as a linear combination of the two possible solutions:

$$
B(q)=A_{1} q^{\beta_{1}}+A_{2} q^{\beta_{2}},
$$

We can simplify this solution using economic theory. In particular, note that as $q$ becomes sufficiently large, the value of the option goes to zero. This implies that

$$
\lim _{q \rightarrow \infty} B(q)=0
$$

Note from equation (ए3) that there will be one positive solution for $\beta$ and one negative solution. Let $\beta_{2}$ denote the positive solution. For this condition to be satisfied, it must be true that $A_{2}=0$. Thus, dropping the subscripts for expositional convenience, this implies that $V\left(q, q^{*}\right)=A q^{\beta}$, where $\beta<0$. 
Furthermore, at the point of exercise, the individual will receive $w / r-F-q^{*} /(r-\mu)$. It follows that $B\left(q=q^{*}\right)=1$. This implies that $A\left(q^{*}\right)^{\beta}=1$, or $A=\left(q^{*}\right)^{-\beta}$. Substituting this into the solution for $B(q)$ yields

$$
B(q)=\left(\frac{q}{q^{*}}\right)^{\beta}
$$

Plugging this into equation (घ) yields equation (『) from the main text. 